\title{
2021 Guidelines for Purchasing and Using Commercial Natural Enemies and Biopesticides in North America ${ }^{1}$
}

\author{
Lynn M. LeBeck and Norman C. Leppla²
}

This guide provides assistance in selecting, purchasing and using commercially available natural enemies and biopesticides for managing accurately diagnosed pest problems. It therefore applies only to situations in which the cause of a pest problem is known and a biological control solution is sought. To choose a commercial natural enemy product, first use Table 1 to locate the habitat of your plant or animal pest and identify the insect or mite. Then, consider using some of the listed types of natural enemies (parasitic nematodes, predatory mites, predatory insects, and parasitic wasps) and biopesticides available to manage these pests. Products often can be used in combination when there is more than one pest problem, and sometimes a product will manage a pest for which it was not intended. The reference numbers correspond with the numbered scientific names of natural enemies in Tables 2-5. The natural enemy source companies identified randomly in Tables $2-5$ are listed in Table 6, along with their websites. Table 7 provides scientific names for some of the most common microbial insecticides and fungicides that can be used to manage many types of pests alone or, if compatible, in combination with insect and mite natural enemies. The title to Table 7 includes a link to the searchable IR-4 Biopesticide and Organic Database for Integrated Pest Management that lists products, sources, and applications. Member practitioners of the
Association of Natural Biocontrol Producers shown in Table 8 provide consulting and other support services that are increasingly important for large-scale implementation of biological control. Sources of information on obtaining and using commercial natural enemies follow in the next section.

Biological control companies typically provide customer service to assure that their products are used appropriately. Information they supply includes the availability and cost of natural enemies and biopesticides, descriptions of individual target pests and their biology, and recommendations for applying and evaluating their products. Product instructions usually indicate the habitats and seasons in which the pests are encountered, developmental stages that are susceptible to parasitism or predation, and relevant behavior of the natural enemies, e.g., how far they move and how many pests they can parasitize or consume. Companies included in the detailed species lists (Tables 2-5) are members of the Association of Natural Biocontrol Producers (ANBP). Producers and distributors belonging to ANBP are preferred because they adhere to a quality assurance policy and code of ethics for the industry and promote research and education on the use of natural enemies. Not listed are most garden centers, companies

1. This document is IPM-146 (originally titled Guidelines for Purchasing and Using Commercial Natural Enemies and Biopesticides in Florida and Other States, published July 2010 by Norman C. Leppla, professor, and Kenneth L. Johnson II, USDA, NIFA, National Needs Fellow, IPM Florida), one of a series of the Entomology and Nematology Department, UF/IFAS Extension. Original publication date July 2010. Revised August 2015 and Februrary 2021. Visit the EDIS website at https://edis.ifas.ufl.edu for the currently supported version of this publication.

2. Lynn M. LeBeck, executive director, Association of Natural Biocontrol Producers (ANBP); and Norman C. Leppla, professor and integrated pest management program director, Entomology and Nematology Department; UF/IFAS Extension, Gainesville, FL 32611.

The use of trade names in this publication is solely for the purpose of providing specific information. UF/IFAS does not guarantee or warranty the products named, and references to them in this publication do not signify our approval to the exclusion of other products of suitable composition.

The Institute of Food and Agricultural Sciences (IFAS) is an Equal Opportunity Institution authorized to provide research, educational information and other services

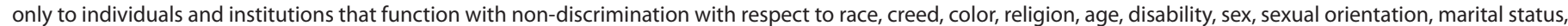

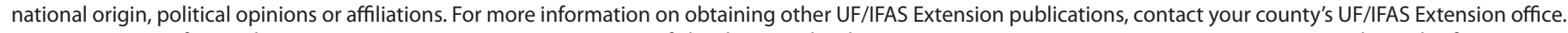
U.S. Department of Agriculture, UF/IFAS Extension Service, University of Florida, IFAS, Florida A \& M University Cooperative Extension Program, and Boards of County Commissioners Cooperating. Nick T. Place, dean for UF/IFAS Extension. 
with very limited geographic markets or product lines, suppliers without comprehensive websites, governmental and other non-commercial producers, so-called big-box stores, and outlets for which information was difficult to find or use.

It is essential to determine that the purchased living organisms are healthy and able to survive long enough to provide biological control in the pest habitat. Suppliers usually provide high-quality natural enemy products but are unable to control conditions during shipment and handling. Temperature extremes, condensation from ice packs, restricted oxygen supply, high organism densities, and long shipping and storage times are some of the factors that can adversely affect natural enemy quality. Therefore, customers should open packages immediately on arrival to provide a better environment for the organisms and detect any potential problems. Packages at least should be inspected for condensation or a fermenting smell, and the number of living and dead organisms should be estimated. If pupae or parasitized host organisms are shipped, the number of emerging adults should be recorded; a sex ratio of at least $40 \%-45 \%$ females is expected. Customers are advised to make sure that most eggs hatch or adults are able to move, if products are shipped in these stages of development. Notes should be made on the product name, company batch number, date received, packaging type and condition, number of organisms in the package, and any other pertinent observations on the appearance and performance of the product. After completing the general check, customers can perform additional quality assurance testing, recording the test methods, number of organisms tested and date, or use the products as soon as possible. An easy-to-use guide, Grower Guide: Quality Assurance of Biocontrol Products, is available to help customers assess the quality of natural enemies received from suppliers (see Buitenhuis 2014 in Sources of Information). The supplier should be notified immediately if there is a problem with the products.

Customers who use biological control products generally want to be directly involved in solving their pest problems. This involvement is essential because products must first be selected and deployed according to general instructions and subsequently evaluated for site-specific effectiveness. It may be necessary to try different products or application procedures, or to modify the environment in ways that enhance the impact of natural enemies. This may involve changing how plants are grown or adding food, companion plants and refuges for natural enemies. The impacts of commercial natural enemies can be limited to the stage that is released or be long-term if they reproduce and become established. Typically, several pests are present, and if some must be managed with pesticides, it is necessary to know which pesticides are compatible with the natural enemies. Other considerations are how to release the natural enemies and in what developmental stages. They can be introduced, for example, on special plants with non-pest hosts (socalled "banker plants") added as eggs, or allowed to fly from release containers. These kinds of considerations may be addressed in instructions from the source companies or gleaned from the references in this guide.

Commercial biological control products described in this guide have been thoroughly tested for effectiveness and given federal and state approval to assure that they can be released into the environment safely. The products are marketed directly by producers or provided by suppliers after obtaining the necessary shipping permits for natural enemies or EPA registrations for biopesticides. Only products (nematodes, mites, and insects) that are insectaryreared, as opposed to field-collected (e.g., lady beetles), and biopesticides that are considered useful and that are available in North America are included. The guide is updated periodically because some products may be discontinued and new ones may become available. ANBP membership companies are contacted directly for their updated information. Specialized products, such as those used for weed management, have been excluded from the guide.

\section{Sources of Information on Obtaining and Using Commercial Natural Enemies and Biopesticides}

Association of Natural Biocontrol Producers (ANBP) Website (http://www.anbp.org). [This is a global commercial biological control organization with members primarily in North America. The website lists producers, distributors, practitioners, and contributing members. Most of the producers and distributors list their products and provide instructions for their use.]

Buitenhuis, R. 2014. Grower Guide: Quality Assurance of Biocontrol Products. Vineland Research and Innovation Centre, Ontario, Canada (https://www.vinelandresearch. com/wp-content/uploads/2020/02/Grower-Guide.pdf) [Procedures are provided for assessing the quality of 28 commercial natural enemies.

Copping, L. G. 2001. The Biopesticide Manual. British Crop Protection Council, $2^{\text {nd }}$ edition. Farnham, UK. 528 p. [This book contains a comprehensive listing and technical descriptions of biopesticides.] 
Electronic Data Information Source (EDIS) website (https://edis.ifas.ufl.edu). [The EDIS website is a comprehensive, single-source repository of all current UF/IFAS numbered peer-reviewed publications. The database is searchable by topic, e.g., agriculture or lawn and garden, and by key words.]

Featured Creatures website (http://entnemdept.ufl.edu/ creatures). [This is a set of in-depth profiles of insects, nematodes, arachnids and other organisms. The database is searchable by common name, scientific name, crop or habitat, higher classification, and recent additions.]

Flint, M. L., S. H. Dreistadt and J. K. Clark. 1998. Natural Enemies Handbook. University of California Integrated Pest Management Project. University of California Press, Los Angeles. 154 p. [This book can be used to identify and use many of the most common natural enemies. It contains a considerable amount of information about biological control, including the toxicity to natural enemies of selected insecticides and acaracides.]

Gerson, U., R. L. Smiley and R. Ochoa. 2003. Mites (Acari) for Pest Control. Wiley-Blackwell. 560 p. [This book describes 34 acarine families that include mites useful for controlling pest mites, insects, nematodes, and weeds. It also contains information on using the mites.]

Hajek, A. E., and J. Eilenberg. 2018. Natural Enemies: An Introduction to Biological Control, 2nd edition. Cambridge University Press. 452 p. [This book describes the diversity of organisms used in biological control.]

Hoffman, M. P., and A. C. Frodsham. 1993. Natural Enemies of Vegetable Insect Pests. Cornell Cooperative Extension, Cornell University, Ithaca, N.Y. 63 p. [This book facilitates identification of the major parasites and predators of insect pests of vegetables. It also contains information on entomopathogenic nematodes and microbial insecticides.]

Hoy, M. A. 2011. Agricultural Acarology, Introduction to Integrated Mite Management. CRC Press. 430 p. [This book contains a general introduction to acarology, including the use of mites for biological control.]

International Biocontrol Manufacturers Association (IBMA) website (https://www.ibma-global.org/). [This is a global commercial biological control organization with members primarily in Europe. It has an Invertebrate Biocontrol Agents (IBCA) Professional Group for producers of macroorganisms (insects, mites and entomopathogenic nematodes).]
International Organization for Biological Control (IOBC) website (https://www.iobc-global.org/). [IOBC promotes the development of biological control and its application in integrated pest management. It has biological control working groups and publications.]

Interregional Research Project No. 4 (IR-4) website (https:// www.ir4project.org). [IR-4 maintains a Biopesticide and Organic Database for Integrated Pest Management (http:// ir4app.rutgers.edu/biopestPub/labelDb.aspx). Search categories include commercial crops, commercial turf and ornamentals, residential food crops, residential turf and ornamentals, pest problems (insects, diseases, weeds, nematodes, and animals), plant growth regulators, and states.]

Lacey, L. 2016. Microbial control of insect and mite pests, from theory to practice. Academic Press. 482 p. (https:// www.elsevier.com/books/microbial-control-of-insect-andmite-pests/lacey/978-0-12-803527-6). [This book describes microbial control agents and their implementation in a variety of crops, along with other applications.]

Malais, M. H. and W. J. Ravensberg. 2003. Knowing and Recognizing (Revised Edition). Koppert Biological Systems. 443 p. (https://www.koppertus.com/knowing-recognizing.). [The Koppert Biological Systems website also has information on the toxicity of selected pesticides to natural enemies.]

\section{Acknowledgments}

The authors thank Dr. Marshall W. Johnson (University of California, Riverside) for a thorough review of the manuscript and Dr. John L. Capinera (University of Florida) for guidance on its structure. Carol S. Glenister (IPM Laboratories) provided an expert review as a producer and supplier of natural enemies. The guide also was reviewed by the Board of Directors of the Association of Natural Biocontrol Producers. This guide was developed in response to requests for assistance in purchasing and using commercial natural enemies. 
Table 1. Habitats of plant or animal pests in North America, typical pests, type of commercial natural enemies available to manage each pest, and species reference number.

\begin{tabular}{|c|c|c|c|}
\hline \multirow{2}{*}{$\begin{array}{l}\text { Habitats of Plant or Animal } \\
\text { Pests }\end{array}$} & \multirow[t]{2}{*}{ Identified Pest } & \multicolumn{2}{|c|}{ Commercial Natural Enemies } \\
\hline & & Type & No. (see Tables 2-5) \\
\hline \multirow[t]{13}{*}{ Citrus } & aphids & predatory insects & $32,34,38-40,42$ \\
\hline & beetles (grubs) & parasitic nematodes & 4,9 \\
\hline & \multirow[t]{2}{*}{ caterpillars } & predatory insects & $37-40,42$ \\
\hline & & parasitic wasps & 65,66 \\
\hline & \multirow[t]{2}{*}{ mealybugs } & predatory insects & $25,27,42$ \\
\hline & & parasitic wasps & 54 \\
\hline & mites & predatory mites & 20,21 \\
\hline & psyllids & parasitic wasps & 57 \\
\hline & \multirow[t]{2}{*}{ scales } & predatory insects & 27 \\
\hline & & parasitic wasps & 45 \\
\hline & \multirow[t]{2}{*}{ thrips } & predatory mites & 17 \\
\hline & & predatory insects & 34,42 \\
\hline & whiteflies & predatory insects & $26,34,38-40,42$ \\
\hline \multirow{22}{*}{$\begin{array}{l}\text { Fruits, Vegetables and Tree } \\
\text { Crops }\end{array}$} & \multirow[t]{2}{*}{ aphids } & predatory insects & $32,34,35,38-42$ \\
\hline & & parasitic wasps & $44,49-51$ \\
\hline & \multirow[t]{3}{*}{ beetles (grubs) } & parasitic nematodes & 2 \\
\hline & & predatory insects & 37 \\
\hline & & parasitic wasps & 56 \\
\hline & \multirow[t]{2}{*}{ caterpillars } & predatory insects & $26,34,35,38-40,42$ \\
\hline & & parasitic wasps & $65,66,69$ \\
\hline & \multirow[t]{2}{*}{ fungus gnats } & predatory mites & 13 \\
\hline & & predatory insects & 31 \\
\hline & leafminers & parasitic wasps & 52,55 \\
\hline & \multirow[t]{2}{*}{ mealybugs } & predatory insects & $25,27,41,42$ \\
\hline & & parasitic wasps & 54 \\
\hline & \multirow[t]{2}{*}{ mites } & predatory mites & $19-24$ \\
\hline & & predatory insects & $28,33,43$ \\
\hline & scales & predatory insects & 27,29 \\
\hline & \multirow[t]{2}{*}{ thrips } & predatory mites & $13,16,20$ \\
\hline & & predatory insects & $31,34,38-40,42$ \\
\hline & \multirow[t]{2}{*}{ whiteflies } & predatory mites & 17 \\
\hline & & predatory insects & $25,34-41$ \\
\hline & Lygus bugs (strawberries) & parasitic wasps & 53 \\
\hline & root-knot nematode (greenhouse tomatoes) & parasitic nematodes & 7 \\
\hline & tomato/potato psyllids & parasitic wasps & 42 \\
\hline
\end{tabular}




\begin{tabular}{|c|c|c|c|}
\hline \multirow{2}{*}{$\begin{array}{c}\text { Habitats of Plant or Animal } \\
\text { Pests }\end{array}$} & \multirow[t]{2}{*}{ Identified Pest } & \multicolumn{2}{|c|}{ Commercial Natural Enemies } \\
\hline & & Type & No. (see Tables 2-5) \\
\hline \multirow{21}{*}{$\begin{array}{l}\text { Ornamental Plants and } \\
\text { Landscapes }\end{array}$} & \multirow[t]{2}{*}{ aphids } & predatory insects & $32,34,35,38-42$ \\
\hline & & parasitic wasps & $44,49-51$ \\
\hline & \multirow[t]{2}{*}{ beetles (grubs) } & parasitic nematodes & $1,2,4,8,12$ \\
\hline & & predatory insects & $37-40$ \\
\hline & caterpillars & parasitic wasps & 65,69 \\
\hline & \multirow[t]{3}{*}{ fungus gnats } & parasitic nematodes & 6,12 \\
\hline & & predatory mites & 13,14 \\
\hline & & predatory insects & 27,31 \\
\hline & \multirow[t]{2}{*}{ leafminers } & parasitic nematodes & 6 \\
\hline & & parasitic wasps & 52,55 \\
\hline & \multirow[t]{3}{*}{ mealybugs } & parasitic nematodes & 1 \\
\hline & & predatory insects & $25,27,41,42$ \\
\hline & & parasitic wasps & 54 \\
\hline & \multirow[t]{2}{*}{ mites } & predatory mites & $19-24$ \\
\hline & & predatory insects & 28,33 \\
\hline & scales & predatory insects & 27,29 \\
\hline & \multirow[t]{3}{*}{ thrips } & parasitic nematodes & 6,12 \\
\hline & & predatory mites & $13,14,16,17,20$ \\
\hline & & predatory insects & $31,34,38-40,42$ \\
\hline & \multirow[t]{2}{*}{ whiteflies } & predatory mites & 17 \\
\hline & & predatory insects & $26,34,38-42$ \\
\hline \multirow{17}{*}{$\begin{array}{l}\text { Greenhouses and } \\
\text { Interiorscapes }\end{array}$} & \multirow[t]{2}{*}{ aphids } & predatory insects & $32,35,41,42$ \\
\hline & & parasitic wasps & $44,46,49-51$ \\
\hline & caterpillars (moth eggs) & parasitic wasps & 65 \\
\hline & \multirow[t]{3}{*}{ fungus gnats } & parasitic nematodes & 5 \\
\hline & & predatory mites & 14 \\
\hline & & predatory insects & 31 \\
\hline & leafminers & parasitic wasps & 52,55 \\
\hline & \multirow[t]{2}{*}{ mealybugs } & predatory insects & $25,41,42$ \\
\hline & & parasitic wasps & 54 \\
\hline & \multirow[t]{2}{*}{ mites } & predatory mites & $19-24$ \\
\hline & & predatory insects & 28,33 \\
\hline & \multirow[t]{2}{*}{ scales } & predatory insects & 30 \\
\hline & & parasitic wasps & 46 \\
\hline & \multirow[t]{2}{*}{ thrips } & predatory mites & $14,18,20$ \\
\hline & & predatory insects & 31 \\
\hline & \multirow[t]{2}{*}{ whiteflies } & predatory insects & $26,35,41,42$ \\
\hline & & parasitic wasps & $46-48$ \\
\hline \multirow[t]{3}{*}{ Turf and Lawns } & caterpillars & parasitic nematodes & 10 \\
\hline & beetles (grubs) & parasitic nematodes & 10,12 \\
\hline & mole crickets & parasitic nematodes & 12 \\
\hline \multirow[t]{2}{*}{ Animal Waste } & \multirow[t]{2}{*}{ filth flies } & predatory insects & 30 \\
\hline & & parasitic wasps & $58-64$ \\
\hline
\end{tabular}


Table 2. Parasitic nematodes. Numbered biological control products [family, genus and species], some (target pests) and source companies.

\begin{tabular}{|c|c|}
\hline \multicolumn{2}{|r|}{ Parasitic Nematodes } \\
\hline Heterorhabditidae & Source Company (Randomized, see Table 7) \\
\hline $\begin{array}{l}\text { Heterorhabditis bacteriophora (cucumber, scarab, Japanese } \\
\text { and flea beetles, thrips, white grubs, corn root worms, } \\
\text { Colorado potato beetles, black vine weevils, and root } \\
\text { mealybugs, on ornamentals, trees and shrubs) }\end{array}$ & $\begin{array}{l}\text { BioWorks } \bullet \text { Crop Defenders } \bullet \text { Bioline AgroSciences } \bullet \text { Buglogical Control } \\
\text { Systems } \bullet \text { Anatis Bioprotection } \bullet \text { Biobest Mexico S.A. de C.V. } \bullet \text { Green Methods } \bullet \\
\text { BioBee } \bullet \text { Evergreen Growers Supply } \bullet \text { Rincon-Vitova } \bullet \text { Natural Enemies } \bullet \text { Sound } \\
\text { Horticulture } \bullet \text { BASF } \bullet \text { ARBICO } \bullet \text { Beneficial Insectary } \bullet \text { Everwood Farm } \bullet \text { Natural } \\
\text { Insect Control } \bullet \text { Sierra Biological } \bullet \text { Biobest } \bullet \text { Koppert } \bullet \text { Plant Products }\end{array}$ \\
\hline $\begin{array}{l}\text { Heterorhabditis megidis (vine weevil larvae on ornamentals, } \\
\text { trees, shrubs and strawberries) }\end{array}$ & Global Horticultural \\
\hline Heterorhabditis indica & Rincon-Vitova • ARBICO \\
\hline $\begin{array}{l}\text { Heterorhabditis spp. (mix) (vine weevil larvae and other soil } \\
\text { borne beetle larvae on ornamentals, trees and shrubs) }\end{array}$ & Dynamic Ecosystems Crop Supply • Natural Insect Control \\
\hline \multicolumn{2}{|l|}{ Steinernematidae } \\
\hline $\begin{array}{l}\text { Steinernema carpocapsae (fungus gnats on potted plants, } \\
\text { wood boring and other caterpillars) }\end{array}$ & $\begin{array}{l}\text { Sierra Biological } \bullet \text { Natural Insect Control } \bullet \text { Natural Enemies } \bullet \text { Buglogical Control } \\
\text { Systems } \bullet \text { BASF } \bullet \text { Koppert } \bullet \text { Rincon-Vitova } \bullet \text { Hydro-Gardens } \bullet \text { Everwood Farm } \\
\cdot \text { Biobest } \bullet \text { Beneficial Insectary } \bullet \text { Green Methods } \bullet \text { Anatis Bioprotection } \bullet \text { Plant } \\
\text { Products } \bullet \text { ARBICO } \bullet \text { Sound Horticulture } \bullet \text { Bioline AgroSciences } \bullet \text { BioBee } \bullet \\
\text { Global Horticultural } \bullet \text { Crop Defenders } \bullet \text { Evergreen Growers Supply }\end{array}$ \\
\hline $\begin{array}{l}\text { Steinernema feltiae (thrips, fungus gnats and leafminers on } \\
\text { ornamentals) }\end{array}$ & $\begin{array}{l}\text { BASF } \bullet \text { Global Horticultural } \bullet \text { BioBee } \bullet \text { Green Methods } \bullet \text { Bioline AgroSciences } \bullet \\
\text { Crop Defenders } \bullet \text { Buglogical Control Systems } \bullet \text { Sierra Biological } \bullet \text { Tip Top Bio- } \\
\text { Control } \bullet \text { Bio-Control, S. A. } \bullet \text { Hydro-Gardens } \bullet \text { Natural Insect Control } \bullet \text { BioWorks } \\
\cdot \text { Koppert } \bullet \text { ARBICO } \bullet \text { Rincon-Vitova } \bullet \text { Beneficial Insectary } \bullet \text { Biobest } \bullet \text { Anatis } \\
\text { Bioprotection } \bullet \text { Orcon } \bullet \text { Biobest Mexico S.A. de C.V. } \bullet \text { Everwood Farm } \bullet \text { Sound } \\
\text { Horticulture } \bullet \text { Plant Products } \bullet \text { Evergreen Growers Supply } \bullet \text { Natural Enemies }\end{array}$ \\
\hline $\begin{array}{l}\text { Steinernema feltiae MG-13 (root-knot nematodes on } \\
\text { greenhouse tomatoes) }\end{array}$ & Sierra Biological \\
\hline Steinernema kraussei (black vine weevil) & $\begin{array}{l}\text { Biobest } \bullet \text { ARBICO } \bullet \text { Rincon-Vitova } \bullet \text { Evergreen Growers Supply } \bullet \text { Beneficial } \\
\text { Insectary } \bullet \text { Sound Horticulture } \bullet \text { Global Horticultural } \bullet \text { BASF } \bullet \text { Bioline } \\
\text { AgroSciences } \bullet \text { Crop Defenders }\end{array}$ \\
\hline $\begin{array}{l}\text { Steinernema riobrave (mole crickets, root weevils on citrus, } \\
\text { other weevils, caterpillars) }\end{array}$ & BASF • ARBICO • Sierra Biological • Sound Horticulture \\
\hline Steinernema spp. (grubs, caterpillars, and fungus gnats) & $\begin{array}{l}\text { Green Methods } \bullet \text { Natural Insect Control } \bullet \text { Bio Control, S.A. • Dynamic } \\
\text { Ecosystems Crop Supply • Crop Defenders }\end{array}$ \\
\hline \multicolumn{2}{|l|}{ Nematode Species Mixes } \\
\hline $\begin{array}{l}\text { Heterorhabditis bacteriophora and Steinernema } \\
\text { carpocapsae (soilborne pests) }\end{array}$ & $\begin{array}{l}\text { Sierra Biological } • \text { Sound Horticulture } \bullet \text { Buglogical Control Systems } • \text { Natural } \\
\text { Insect Control }\end{array}$ \\
\hline $\begin{array}{l}\text { Steinernema feltiae and Heterorhabditis spp. (soilborne } \\
\text { pests) }\end{array}$ & $\begin{array}{l}\text { Buglogical Control Systems } \bullet \text { Hydro-Gardens } \bullet \text { Anatis Bioprotection } \bullet \text { Dynamic } \\
\text { Ecosystems Crop Supply } \bullet \text { Natural Insect Control } \bullet \text { Sound Horticulture } \bullet \text { Crop } \\
\text { Defenders }\end{array}$ \\
\hline
\end{tabular}


Table 3. Predatory mites. Numbered biological control products [family, genus and species], some (target pests) and source companies.

\begin{tabular}{|c|c|}
\hline \multicolumn{2}{|r|}{ Predatory Mites } \\
\hline Laelapidae & Source Company (Randomized, see Table 7) \\
\hline $\begin{array}{l}\text { Hypoaspis (Gaeolaelaps) gillespieii } \\
\text { (fungus gnats, thrips) }\end{array}$ & $\begin{array}{l}\text { Dynamic Ecosystems Crop Supply } \bullet \text { Crop Defenders } \bullet \text { Anatis Bioprotection } \bullet \text { Natural Insect Control } \bullet \\
\text { Applied Bio-nomics } \bullet \text { WestGrow Biological Solutions }\end{array}$ \\
\hline $\begin{array}{l}\text { Stratiolaelaps scimitus (also } \\
\text { called Hypoaspis or Stratiolaelaps } \\
\text { miles) (fungus gnats and thrips on } \\
\text { potted plants, bedding plants and } \\
\text { seedlings) }\end{array}$ & $\begin{array}{l}\text { BioBee } \bullet \text { WestGrow Biological Solutions } \bullet \text { Plant Products } \bullet \text { Sound Horticulture } \bullet \text { Evergreen Growers } \\
\text { Supply } \bullet \text { GrowLiv Biologicals } \bullet \text { Natural Insect Control } \bullet \text { Beneficial Insectary } \bullet \text { Everwood Farm } \bullet \text { IPM } \\
\text { Laboratories } \bullet \text { Natural Enemies } \bullet \text { Green Methods } \bullet \text { Global Horticultural } \bullet \text { Crop Defenders } \bullet \text { Dynamic } \\
\text { Ecosystems Crop Supply } \bullet \text { Buglogical Control Systems } \bullet \text { Bioline AgroSciences } \bullet \text { Tip Top Bio-Control } \bullet \\
\text { Anatis Bioprotection } \bullet \text { Rincon-Vitova } \bullet \text { Biobest } \bullet \text { Applied Bio-nomics } \bullet \text { Koppert } \bullet \text { ARBICO } \bullet \text { Bio Control, } \\
\text { S.A. }\end{array}$ \\
\hline
\end{tabular}

\section{Phytoseiidae}

Amblyseius andersoni (spider, russet, rust and broad mite)

Everwood Farm • Green Methods • Natural Enemies • Global Horticultural • Hydro-Gardens • RinconVitova $\bullet$ Bioline AgroSciences $•$ ARBICO • Biobest • Tip Top Bio-Control • Sound Horticulture • Evergreen Growers Supply $\bullet$ Crop Defenders $•$ Plant Products • Beneficial Insectary • Natural Insect Control •IPM Laboratories $\bullet$ BioBee

Amblyseius degenerans (also called Beneficial Insectary • Plant Products • Bio Control, S.A. • Natural Insect Control • Biobest Mexico S.A. de Iphiseius degenerans) (thrips, broad and spider mites on peppers and ornamentals)

Amblyseius swirskii (whiteflies and thrips on vegetables, melons and ornamentals)

\section{Amblydromalus limonicus}

(formerly called Typhlodromalus limonicus) (thrips and whiteflies in greenhouses)

Neoseiulus californicus (formerly called Amblyseius californicus) (twospotted spider, broad and cyclamen mites on ornamentals, vegetables, fruit and potted plants)

Neoseiulus cucumeris (formerly called Amblyseius cucumeris) (twospotted spider mite and tarsonemid mites, and flower thrips on all crops)

Neoseiulus fallacis (formerly called Amblyseius fallacis) (two-spotted spider, European red, and citrus red mites on many crops)

Galendromus occidentalis (spider, eriophyid and russet mites on ornamentals and vegetables in greenhouses and interiorscapes)

Mesoseiulus longipes (also called Phytoseiulus longipes) (two spotted spider mites in greenhouses and interiorscapes)
Beneficial Insectary $\bullet$ Plant Products $\bullet$ Bio Control, S.A. $\bullet$ Natural Insect Con
C.V. $\bullet$ Sound Horticulture $\bullet$ Biobest $\bullet$ Crop Defenders $\bullet$ Global Horticultural

BioBee • Evergreen Growers Supply • Biobest • Natural Insect Control • ARBICO • Green Methods • Biobest Mexico S.A. de C.V. $\bullet$ Crop Defenders $\bullet$ Rincon-Vitova $\bullet$ Buglogical Control Systems $\bullet$ Biotactics $\bullet$ Tip Top Bio-Control • Bioline AgroSciences $\bullet$ Hydro-Gardens $\bullet$ Beneficial Insectary $\bullet$ Global Horticultural - Koppert • Bio Control, S.A. • Everwood Farm • GrowLiv Biologicals • Sound Horticulture • Dynamic Ecosystems Crop Supply • Natural Enemies • Plant Products

Koppert • Everwood Farm

Associates Insectary • Beneficial Insectary $\bullet$ Hydro-Gardens $\bullet$ Koppert $\bullet$ Buglogical Control Systems - Crop Defenders • Foothill Agricultural Research • Bio Control, S.A. • Plant Products • Bioline AgroSciences • ARBICO • Biobest Mexico S.A. de C.V. • Evergreen Growers Supply • Sound Horticulture - Biotactics $\bullet$ Rincon-Vitova $\bullet$ Everwood Farm • Biobest $•$ BioBee $•$ Natural Insect Control $•$ Tip Top Bio-Control • Green Methods •IPM Laboratories • Orcon • Global Horticultural • Natural Enemies • WestGrow Biological Solutions • Dynamic Ecosystems Crop Supply • GrowLiv Biologicals

Tip Top Bio-Control • Bio Control, S.A. • Koppert • Rincon-Vitova • Dynamic Ecosystems Crop Supply - Biobest Mexico S.A. de C.V. • Anatis Bioprotection • Natural Insect Control • Applied Bio-nomics - Hydro-Gardens • Beneficial Insectary • Plant Products • Sound Horticulture $\bullet$ Crop Defenders • Buglogical Control Systems •IPM Laboratories • Global Horticultural • GrowLiv Biologicals • Biobest • Natural Enemies • Everwood Farm • ARBICO • Evergreen Growers Supply • BioBee $\bullet$ Green Methods • Bioline AgroSciences • Orcon

Biotactics • Beneficial Insectary •Tip Top Bio-Control • Everwood Farm • Applied Bio-nomics • Dynamic Ecosystems Crop Supply • Anatis Bioprotection • Rincon-Vitova • Sound Horticulture • ARBICO • Green Methods $•$ Crop Defenders $•$ Natural Enemies $•$ Buglogical Control Systems • Evergreen Growers Supply - Natural Insect Control • WestGrow Biological Solutions • Plant Products

Foothill Agricultural Research • ARBICO • Evergreen Growers Supply • Buglogical Control Systems • Biotactics $\bullet$ Hydro-Gardens $\bullet$ Natural Insect Control • Rincon-Vitova $\bullet$ Sound Horticulture

Natural Insect Control • Hydro-Gardens • Evergreen Growers Supply • Sound Horticulture • Buglogical Control Systems • IPM Laboratories • ARBICO • Rincon-Vitova • Tip Top Bio-Control • Biotactics 


\section{Predatory Mites}

Laelapidae

Phytoseiulus persimilis (spider mites on many crops)

\section{Source Company (Randomized, see Table 7)}

Anatis Bioprotection • Plant Products $\bullet$ Green Methods $\bullet$ Biotactics $\bullet$ BioBee $\bullet$ Koppert $\bullet$ Biobest $\bullet$ Rincon-Vitova $\bullet$ Hydro-Gardens • Orcon • Applied Bio-nomics • Buglogical Control Systems • Natural Enemies • Everwood Farm • Crop Defenders • Sound Horticulture • Biobest Mexico S.A. de C.V. • ARBICO -WestGrow Biological Solutions • Evergreen Growers Supply • Bioline AgroSciences • Bio Control, S.A. • Beneficial Insectary • Natural Insect Control • Dynamic Ecosystems Crop Supply • Global Horticultural • GrowLiv Biologicals

Table 4. Predatory insects. Numbered biological control products [family, genus and species], some target pests and source companies.

\begin{tabular}{|c|c|}
\hline \multicolumn{2}{|c|}{ Predatory Insects } \\
\hline & Source Company (Randomized, see Table 7) \\
\hline \multicolumn{2}{|r|}{ Coleoptera } \\
\hline \multicolumn{2}{|l|}{ Coccinellidae } \\
\hline $\begin{array}{l}\text { Cryptolaemus montrouzieri mealybug destroyer (mealybugs } \\
\text { on citrus, ornamentals, and vegetables, including greenhouses } \\
\text { and interiorscapes) }\end{array}$ & $\begin{array}{l}\text { Crop Defenders } \bullet \text { IPM Laboratories } \bullet \text { Evergreen Growers Supply } \bullet \text { Beneficial } \\
\text { Insectary } \bullet \text { Associates Insectary } \bullet \text { Hydro-Gardens } \bullet \text { BioBee } \bullet \text { Global } \\
\text { Horticultural } \bullet \text { Buglogical Control Systems } \bullet \text { Bio Control, S.A. } \bullet \text { Foothill } \\
\text { Agricultural Research } \bullet \text { Sound Horticulture } \bullet \text { Biobest } \bullet \text { Everwood Farm } \bullet \\
\text { Green Methods } \bullet \text { Koppert } \bullet \text { Plant Products } \bullet \text { Natural Insect Control } \bullet \text { Anatis } \\
\text { Bioprotection } \bullet \text { Orcon } \bullet \text { Bioline AgroSciences } \bullet \text { Rincon-Vitova } \bullet \text { Biobest Mexico } \\
\text { S.A. de C.V. } \bullet \text { Tip Top Bio-Control } \bullet \text { ARBICO }\end{array}$ \\
\hline $\begin{array}{l}\text { Delphastus catalinae (formerly called Delphastus pusillus) } \\
\text { whitefly predator (greenhouse, banded-winged, sweetpotato, } \\
\text { woolly, azalea, hibiscus, cloudywinged, citrus and } \\
\text { rhododendron whiteflies on ornamentals, vegetables, fruit, } \\
\text { and citrus, including greenhouses and interiorscapes) }\end{array}$ & $\begin{array}{l}\text { Orcon } \bullet \text { WestGrow Biological Solutions } \bullet \text { Crop Defenders } \bullet \text { Anatis } \\
\text { Bioprotection } \bullet \text { Applied Bio-nomics } \bullet \text { Buglogical Control Systems } \bullet \text { Dynamic } \\
\text { Ecosystems Crop Supply } \bullet \text { Natural Enemies } \bullet \text { Koppert } \bullet \text { Sound Horticulture } \bullet \\
\text { ARBICO } \bullet \text { Natural Insect Control } \bullet \text { Evergreen Growers Supply } \bullet \text { Rincon-Vitova } \\
\cdot \text { Plant Products } \bullet \text { IPM Laboratories } \bullet \text { Hydro-Gardens } \bullet \text { Beneficial Insectary } \bullet \\
\text { BioBee }\end{array}$ \\
\hline $\begin{array}{l}\text { Rhyzobius lophanthae (also called Lindorus lophanthae) (hard } \\
\text { and soft scales and mealybugs on ornamentals, vegetables, } \\
\text { citrus and fruit) }\end{array}$ & $\begin{array}{l}\text { Evergreen Growers Supply } \bullet \text { Rincon-Vitova } \bullet \text { Anatis Bioprotection } \bullet \text { Buglogical } \\
\text { Control Systems } \bullet \text { IPM Laboratories } \bullet \text { ARBICO } \bullet \text { Natural Insect Control } \bullet \\
\text { Foothill Agricultural Research }\end{array}$ \\
\hline Stethorus punctillum (two-spotted spider mites) & $\begin{array}{l}\text { Applied Bio-nomics } \bullet \text { BioBee } \bullet \text { Natural Insect Control } \bullet \text { Crop Defenders } \bullet \\
\text { Sound Horticulture } \bullet \text { Dynamic Ecosystems Crop Supply } \bullet \text { ARBICO } \bullet \text { Anatis } \\
\text { Bioprotection } \bullet \text { Buglogical Control Systems } \bullet \text { Natural Enemies } \bullet \text { Evergreen } \\
\text { Growers Supply } \bullet \text { IPM Laboratories } \bullet \text { Rincon-Vitova }\end{array}$ \\
\hline \multicolumn{2}{|l|}{ Cybocephalidae } \\
\hline $\begin{array}{l}\text { Cybocephalus nipponicus scale picnic beetle (euonymus and } \\
\text { San Jose scale on ornamentals, vegetables and fruit, including } \\
\text { greenhouses and interiorscapes) }\end{array}$ & Crop Defenders \\
\hline \multicolumn{2}{|l|}{ Histeridae } \\
\hline Carcinops pumilio (flies in manure) & IPM Laboratories \\
\hline \multicolumn{2}{|l|}{ Staphylinidae } \\
\hline $\begin{array}{l}\text { Dalotia coriaria (also called Atheta coriaria) (fungus gnats, } \\
\text { shore flies and thrips in vegetables and ornamentals, including } \\
\text { greenhouses and interiorscapes) }\end{array}$ & $\begin{array}{l}\text { Plant Products } \bullet \text { Crop Defenders } \bullet \text { ARBICO } \bullet \text { Evergreen Growers Supply } \bullet \text { Tip } \\
\text { Top Bio-Control } \bullet \text { Sound Horticulture } \bullet \text { Global Horticulture } \bullet \text { Natural Insect } \\
\text { Control } \bullet \text { GrowLiv Biologicals } \bullet \text { Rincon-Vitova } \bullet \text { Biobest } \bullet \text { WestGrow Biological } \\
\text { Solutions } \bullet \text { Natural Enemies } \bullet \text { Bioline AgroSciences } \bullet \text { Bio Control, S.A. } \bullet \\
\text { Green Methods } \bullet \text { Applied Bio-nomics } \bullet \text { Dynamic Ecosystems Crop Supply } \\
\cdot \text { Beneficial Insectary } \bullet \text { Buglogical Control Systems } \bullet \text { Anatis Bioprotection } \bullet \\
\text { Everwood Farm } \bullet \text { BioBee }\end{array}$ \\
\hline
\end{tabular}




\section{Predatory Insects}

Source Company (Randomized, see Table 7)

\section{Cecidomyiidae}

Aphidoletes aphidimyza (aphids in citrus, ornamentals, fruits and vegetables, including greenhouses and interiorscapes)

Feltiella acarisuga (spider mites in ornamentals and vegetables, including greenhouses and interiorscapes)

\section{Diptera}

Koppert • Global Horticulture - Anatis Bioprotection $\bullet$ Rincon-Vitova • Natural Enemies • Applied Bio-nomics • ARBICO • BioBee • Green Methods • Buglogical Control Systems • Bioline AgroSciences • Natural Insect Control • Beneficial Insectary • Everwood Farm • Biobest • Evergreen Growers Supply -WestGrow Biological Solutions • Sound Horticulture • Tip Top Bio-Control - Biobest Mexico S.A. de C.V. • Orcon • Bio Control, S.A. • IPM Laboratories • Hydro-Gardens $\bullet$ Crop Defenders $•$ Dynamic Ecosystems Crop Supply • Plant Products

Biobest Mexico S.A. de C.V. • Beneficial Insectary • Rincon-Vitova $\bullet$ Global Horticultural $\bullet$ Koppert $\bullet$ ARBICO $\bullet$ Hydro-Gardens $\bullet$ Biobest $\bullet$ Bio Control, S.A. - Crop Defenders • Sound Horticulture • Plant Products • Natural Enemies • Everwood Farm • Natural Insect Control • Buglogical Control Systems

\section{Hemiptera}

\section{Anthocoridae}

Orius insidiosus minute pirate bug (thrips, aphids and whiteflies on ornamentals, vegetables and citrus, including greenhouses and interiorscapes)
Sound Horticulture $\bullet$ Bio Control, S.A. • Dynamic Ecosystems Crop Supply - Natural Enemies • Natural Insect Control • Bioline AgroSciences • Tip Top Bio-Control • Evergreen Growers Supply • Everwood Farm • Biobest • Buglogical Control Systems • Biobest Mexico S.A. de C.V. • Rincon-Vitova • IPM Laboratories $\bullet$ Beneficial Insectary $\bullet$ Koppert $\bullet$ Crop Defenders $\bullet$ GrowLiv Biologicals • Anatis Bioprotection • ARBICO • Plant Products $\bullet$ BioBee

\section{Reduviidae}

Zelus renardii assassin bug (generalist predator) ARBICO

\section{Miridae}

Dicyphus hesperus (greenhouse and tobacco whiteflies)

Bioline AgroSciences • Biobest • Sound Horticulture $\bullet$ Crop Defenders • Natural Insect Control • ARBICO • Anatis Bioprotection • GrowLiv Biologicals • BioBee

\section{Pentatomidae}

Podisus maculiventris spined soldier bug (Colorado potato beetles and caterpillars on ornamentals, vegetables and citrus)

\section{Chrysopidae}

Chrysoperla carnea green lacewing (aphids and other small soft-bodied insects on ornamentals, citrus, fruit and vegetables)

Chrysoperla rufilabris green lacewing (aphids and other small soft-bodied insects on ornamentals, citrus, fruit and vegetables)

Chrysoperla spp. lacewing (aphids and other small softbodied insects on ornamentals, citrus, fruit and vegetables)
ARBICO • Bioline AgroSciences • Sound Horticulture • Rincon-Vitova • Evergreen Growers Supply • Buglogical Control Systems

\section{Neuroptera}

Beneficial Insectary $\bullet$ BioBee $\bullet$ Plant Products $\bullet$ Crop Defenders $\bullet$ Natural Insect Control • Biobest • Bio Control, S.A. • GrowLiv Biologicals • Buglogical Control Systems • Everwood Farm • Anatis Bioprotection • Orcon • Koppert

Evergreen Growers Supply • Global Horticultural • Natural Enemies • Buglogical Control Systems • Everwood Farm • Plant Products • Sound Horticulture •IPM Laboratories $\bullet$ BioBee $\bullet$ Rincon-Vitova $\bullet$ Natural Insect Control

Biobest • Crop Defenders • ARBICO • Bioline AgroSciences $•$ Kunafin • Natural Insect Control

\section{Hemerobiidae}

Micromus variegatus brown lacewing (aphids, whiteflies and mealybugs on vegetables and ornamentals)

Sympherobius barberi brown lacewing (mealybugs, psyllids, thrips, mites, whiteflies, aphids, small caterpillars, leafhoppers, and insect eggs on grapes, citrus, tree crops and greenhouse crops)
Everwood Farm • Applied Bio-nomics $\bullet$ Crop Defenders $\bullet$ Natural Insect Control • Anatis Bioprotection •WestGrow Biological Solutions • Evergreen Growers Supply • Dynamic Ecosystems Crop Supply

Foothill Agricultural Research 
Source Company (Randomized, see Table 7)

\section{Thysanoptera}

\section{Thripidae}

Scolothrips sexmaculatus (spider mites on fruit trees)

Rincon-Vitova

Table 5. Parasitic wasps. Numbered biological control products [family, genus and species], some (target pests) and source companies.

\begin{tabular}{|c|c|}
\hline \multicolumn{2}{|c|}{ Parasitic Wasps } \\
\hline & Source Company (Randomized, see Table 7) \\
\hline \multicolumn{2}{|c|}{ Hymenoptera } \\
\hline \multicolumn{2}{|l|}{ Aphelinidae } \\
\hline Aphytis melinus (oleander, citrus scales, including greenhouses) & $\begin{array}{l}\text { Hydro-Gardens } \bullet \text { Natural Insect Control } \bullet \text { IPM Laboratories } \bullet \text { Anatis } \\
\text { Bioprotection } \bullet \text { Buglogical Control Systems } \bullet \text { Orcon } \bullet \text { Rincon-Vitova } \\
\cdot \text { Tip Top Bio-Control } \bullet \text { Sound Horticulture } \bullet \text { Foothill Agricultural } \\
\text { Research } \bullet \text { Plant Products } \bullet \text { Evergreen Growers Supply } \bullet \text { ARBICO } \bullet \\
\text { Associates Insectary } \bullet \text { Green Methods }\end{array}$ \\
\hline Encarsia formosa (whiteflies in greenhouses) & $\begin{array}{l}\text { Koppert } \bullet \text { Global Horticultural } \bullet \text { Biobest } \bullet \text { Natural Insect Control } \bullet \\
\text { IPM Laboratories } \bullet \text { Natural Enemies } \bullet \text { Evergreen Growers Supply } \bullet \\
\text { Crop Defenders } \bullet \text { Everwood Farm } \bullet \text { Anatis Bioprotection } \bullet \text { Orcon } \\
\cdot \text { Bio Control, S.A. } \bullet \text { Buglogical Control Systems } \bullet \text { Rincon-Vitova } \bullet \\
\text { Green Methods } \bullet \text { ARBICO } \bullet \text { Beneficial Insectary } \bullet \text { Hydro-Gardens } \bullet \\
\text { Dynamic Ecosystems Crop Supply } \bullet \text { Applied Bio-nomics } \bullet \text { Sound } \\
\text { Horticulture } \bullet \text { Bioline AgroSciences } \bullet \text { WestGrow Biological Solutions } \\
\cdot \text { BioBee } \bullet \text { Plant Products }\end{array}$ \\
\hline $\begin{array}{l}\text { Eretmocerus eremicus (sweetpotato whiteflies, including greenhouses } \\
\text { and interiorscapes) }\end{array}$ & $\begin{array}{l}\text { BioBee } \bullet \text { Global Horticultural } \bullet \text { Beneficial Insectary } \bullet \text { Sound } \\
\text { Horticulture } \bullet \text { ARBICO } \bullet \text { Green Methods } \bullet \text { Buglogical Control } \\
\text { Systems } \bullet \text { Biobest Mexico S.A. de C.V. } \bullet \text { IPM Laboratories } \bullet \text { Biobest } \\
\cdot \text { Bioline AgroSciences } \bullet \text { Natural Insect Control } \bullet \text { Koppert } \bullet \text { Rincon- } \\
\text { Vitova } \bullet \text { Plant Products } \bullet \text { Bio Control, S.A. } \bullet \text { Evergreen Growers } \\
\text { Supply } \bullet \text { Hydro-Gardens } \bullet \text { Crop Defenders } \bullet \text { Everwood Farm }\end{array}$ \\
\hline $\begin{array}{l}\text { Eretmocerus mundus (sweetpotato and tobacco whiteflies in } \\
\text { greenhouses) }\end{array}$ & Bio Control, S.A. • Global Horticultural \\
\hline \multicolumn{2}{|l|}{ Aphidiidae } \\
\hline $\begin{array}{l}\text { Aphidius colemani (cotton, melon, green peach and other aphids } \\
\text { on vegetables, fruit and ornamentals, including greenhouses and } \\
\text { interiorscapes) }\end{array}$ & $\begin{array}{l}\text { GrowLiv Biologicals } \bullet \text { Sound Horticulture } \bullet \text { Everwood Farm } \bullet \text { Global } \\
\text { Horticultural } \bullet \text { Natural Insect Control } \bullet \text { Biobest Mexico S.A. de C.V. } \\
\bullet \text { IPM Laboratories } \bullet \text { Bio Control, S.A. } \bullet \text { Anatis Bioprotection } \bullet \text { Green } \\
\text { Methods } \bullet \text { Tip Top Bio-Control } \bullet \text { Beneficial Insectary } \bullet \text { BioBee } \bullet \\
\text { Evergreen Growers Supply } \bullet \text { Rincon-Vitova } \bullet \text { Buglogical Control } \\
\text { Systems } \bullet \text { ARBICO } \bullet \text { Koppert } \bullet \text { Hydro-Gardens } \bullet \text { Biobest } \bullet \text { Bioline } \\
\text { AgroSciences } \bullet \text { Plant Products } \bullet \text { Crop Defenders } \bullet \text { Natural Enemies }\end{array}$ \\
\hline $\begin{array}{l}\text { Aphidius ervi (potato, pea, and green peach aphids on vegetables, fruit } \\
\text { and ornamentals, including greenhouses and interiorscapes) }\end{array}$ & $\begin{array}{l}\text { Green Methods } \bullet \text { Crop Defenders } \bullet \text { Buglogical Control Systems } \bullet \\
\text { Plant Products } \bullet \text { Biobest Mexico S.A. de C.V. } \bullet \text { Beneficial Insectary } \\
\bullet \text { Bioline AgroSciences } \bullet \text { Rincon-Vitova } \bullet \text { BioBee } \bullet \text { Tip Top Bio- } \\
\text { Control } \bullet \text { Hydro-Gardens } \bullet \text { Global Horticultural } \bullet \text { ARBICO } \bullet \text { Biobest } \\
\text { - Everwood Farm } \bullet \text { Koppert } \bullet \text { Evergreen Growers Supply } \bullet \text { Natural } \\
\text { Insect Control } \bullet \text { IPM Laboratories } \bullet \text { Bio Control, S.A. } \bullet \text { Natural } \\
\text { Enemies } \bullet \text { Global Horticultural } \bullet \text { Dynamic Ecosystems Crop Supply }\end{array}$ \\
\hline $\begin{array}{l}\text { Aphidius matricariae (green peach aphids on vegetables, fruit and } \\
\text { ornamentals, including greenhouses and interiorscapes) }\end{array}$ & $\begin{array}{l}\text { Beneficial Insectary } \bullet \text { Natural Insect Control } \bullet \text { Biobest } \bullet \text { Bio Control, } \\
\text { S.A. } \bullet \text { Evergreen Growers Supply } \bullet \text { Crop Defenders } \bullet \text { ARBICO } \bullet \text { Global } \\
\text { Horticultural }\end{array}$ \\
\hline \multicolumn{2}{|l|}{ Braconidae } \\
\hline $\begin{array}{l}\text { Dacnusa sibirica (leafminers on vegetables, fruit and ornamentals, } \\
\text { including greenhouse and interiorscapes) }\end{array}$ & $\begin{array}{l}\text { Beneficial Insectary } \bullet \text { Natural Insect Control } \bullet \text { Biobest } \bullet \text { Bio Control, } \\
\text { S.A. } \bullet \text { Evergreen Growers Supply } \bullet \text { Crop Defenders } \bullet \text { ARBICO } \bullet \text { Global } \\
\text { Horticultural }\end{array}$ \\
\hline $\begin{array}{l}\text { Peristenus relictus (also called Peristenus relictus and digoneutis), (Lygus } \\
\text { bugs on strawberries) }\end{array}$ & Rincon-Vitova \\
\hline
\end{tabular}




\section{Encyrtidae}

Anagyrus pseudococci (new name is Anagyrus vladimiri) (mealybugs on grapes and citrus)

\section{Eulophidae}

Diglyphus isaea (vegetable, tomato, serpentine and chrysanthemum leafminers on vegetables, fruit and ornamentals, and in greenhouses and interiorscapes)

Pediobius foveolatus (Mexican bean beetle on vegetables)

Tamarixia radiata (Asian citrus psyllid)

\section{Pteromalidae}

Muscidifurax raptor (flies in garbage, manure and compost)

Muscidifurax raptorellus (flies in garbage, manure and compost)

Muscidifurax zaraptor (flies in garbage, manure and compost)

Muscidifurax spp. (flies in garbage, manure and compost)

Nasonia vitripennis (flies in garbage, manure and compost)

Spalangia cameroni (flies in garbage, manure and compost)

Spalangia endius (flies in garbage, manure and compost)

\section{Trichogrammatidae}

Trichogramma brassicae (moth eggs on vegetables, fruit, citrus and ornamentals, including greenhouse and interiorscapes)
Source Company (Randomized, see Table 7)

Foothill Agricultural Research $\bullet$ Rincon-Vitova $\bullet$ Sound Horticulture - ARBICO • BioBee • Associates Insectary • Biobest • Evergreen Growers Supply $\bullet$ Koppert

Biobest • Plant Products $\bullet$ Global Horticultural • Biobest Mexico S.A. de C.V. • BioBee • Bio Control, S.A. • Tip Top Bio-Control • Sound Horticulture $\bullet$ Rincon-Vitova $\bullet$ Natural Insect Control $\bullet$ Bioline AgroSciences $\bullet$ Buglogical Control Systems • Koppert • Beneficial Insectary • Evergreen Growers Supply • Green Methods • ARBICO • Hydro-Gardens $\bullet$ Crop Defenders

Buglogical Control Systems • Natural Insect Control • Sound Horticulture $\bullet$ Rincon-Vitova

Foothill Agricultural Research • Associates Insectary

Spalding Laboratories • Evergreen Growers Supply • Rincon-Vitova - Buglogical Control Systems • Natural Insect Control • Sound Horticulture •IPM Laboratories • Kunafin

IPM Laboratories • Buglogical Control Systems • Spalding Laboratories • Plant Products • Kunafin • Evergreen Growers Supply - Natural Insect Control • Rincon-Vitova • Koppert

Evergreen Growers Supply • Buglogical Control Systems • RinconVitova $\bullet$ Kunafin $\bullet$ Plant Products

Kunafin • Natural Insect Control • Tip Top Bio-Control • Sound Horticulture • Evergreen Growers Supply • Everwood Farm

Buglogical Control Systems

Spalding Laboratories • Rincon-Vitova • Buglogical Control Systems - Plant Products • Kunafin

Kunafin • Spalding Laboratories

Everwood Farm • Tip Top Bio-Control • Natural Insect Control • Orcon • Anatis Bioprotection • Global Horticultural • Beneficial Insectary •IPM Laboratories $\bullet$ Kunafin • ARBICO • Green Methods - Sound Horticulture $\bullet$ Plant Products $\bullet$ Rincon-Vitova $\bullet$ Biobest $\bullet$ Evergreen Growers Supply $\bullet$ Crop Defenders $\bullet$ Buglogical Control Systems

Buglogical Control Systems $\bullet$ Green Methods $•$ Anatis Bioprotection - Crop Defenders • Rincon-Vitova • Orcon • Tip Top Bio-Control • Sound Horticulture $\bullet$ Evergreen Growers Supply $\bullet$ ARBICO • Natural Insect Control • Plant Products • Global Horticultural

Natural Insect Control • Anatis Bioprotection • Plant Products

Trichogramma ostriniae (European corn borer on corn and peppers, and grape berry moth on grapes)

Trichogramma platneri (moth eggs in fruit trees in western US orchards)

Sound Horticulture $\bullet$ Crop Defenders • ARBICO • Evergreen Grower Supply • Rincon-Vitova • Buglogical Control Systems • Everwood Farm • Foothill Agricultural Research • Natural Insect Control • Tip Top Bio-Control

Trichogramma pretiosum (moth eggs on vegetables, field crops and ornamentals)

Plant Products $\bullet$ Crop Defenders $•$ ARBICO $•$ Orcon $•$ Sound Horticulture $\bullet$ Rincon-Vitova $\bullet$ Natural Insect Control $\bullet$ Buglogical Control Systems • Everwood Farm • Green Methods • Bio Control, S.A. • Tip Top Bio-Control

Plant Products • Kunafin

Trichogramma species (moth eggs on vegetables, field crops and ornamentals) 
Table 6. Member companies of the Association of Natural Biocontrol Producers that market nematodes, mites, and insects for pest management in North America. Products available from these companies are listed in Tables 2-5. For companies that produce or sell biopesticides, visit the IR-4 Biopesticide and Organic Database for Integrated Pest Management (http://ir4app.rutgers.edu/ biopestPub/labelDb.aspx).

\begin{tabular}{|c|c|}
\hline Company & Website \\
\hline Anatis Bioprotection & http://anatisbioprotection.com/en/ \\
\hline Applied Bio-nomics & http://www.appliedbio-nomics.com \\
\hline ARBICO Organics & http://www.arbico-organics.com \\
\hline Associates Insectary & http://www.associatesinsectary.com \\
\hline BASF Agricultural Specialties & https://agriculture.basf.com/us/en.html \\
\hline Beneficial Insectary & http://www.insectary.com \\
\hline BioBee USA & http://www.biobee.com/ \\
\hline Biobest Canada & http://www.biobestgroup.com \\
\hline Biobest Mexico & http://www.biobestgroup.com \\
\hline Biobest USA & http://www.biobestgroup.com \\
\hline Bio Control, S. A. & http://biocontrol.cr/ \\
\hline Bioline AgroSciences & https://www.biolineagrosciences.com/ \\
\hline Biotactics & http://www.benemite.com \\
\hline BioWorks & http://www.bioworksinc.com \\
\hline Buglogical Control Systems & http://www.buglogical.com \\
\hline Crop Defenders & http://www.cropdefenders.com/ \\
\hline Dynamic Ecosystems Crop Supply & http://www.dynamicecosystems.ca \\
\hline Evergreen Growers Supply & http://www.evergreengrowers.com \\
\hline Everwood Farm & http://www.everwoodfarm.com/ \\
\hline Foothill Agriculture Research (FAR Inc.) & https://www.far-inc.com/ \\
\hline Global Horticultural & http://www.globalhort.com/ \\
\hline Green Methods & http://greenmethods.com \\
\hline GrowLiv & http://www.growliv.com/ \\
\hline Hydro-Gardens & http://www.hydro-gardens.com \\
\hline IPM Laboratories & http://www.ipmlabs.com \\
\hline Koppert Biological Systems & http://www.koppert.com \\
\hline Kunafin "The Insectary" & http://www.kunafin.com \\
\hline Natural Enemies & https://naturalenemies.com/ \\
\hline Natural Insect Control & http://www.naturalinsectcontrol.com/ \\
\hline Orcon (Organic Control) & http://organiccontrol.com/ \\
\hline Plant Products & https://www.plantproducts.com/ \\
\hline Rincon-Vitova & http://www.rinconvitova.com \\
\hline Sierra Biological Inc. & http://www.sierrabiological.com/ \\
\hline Sound Horticulture & http://soundhorticulture.com/ \\
\hline Spalding Laboratories & https://www.spalding-labs.com \\
\hline Tip Top Bio-Control & http://www.tiptopbiocontrol.com/ \\
\hline WestGrow Biological Solutions & https://www.thebuglady.ca/ \\
\hline
\end{tabular}


Table 7. Biopesticides. Common microbial insecticide and fungicide active ingredients and some target pests. The searchable IR-4 Project Biopesticide and Organic Database for Integrated Pest Management lists the products, sources, and applications for biopesticides (http://ir4app.rutgers.edu/biopestPub/labelDb.aspx).

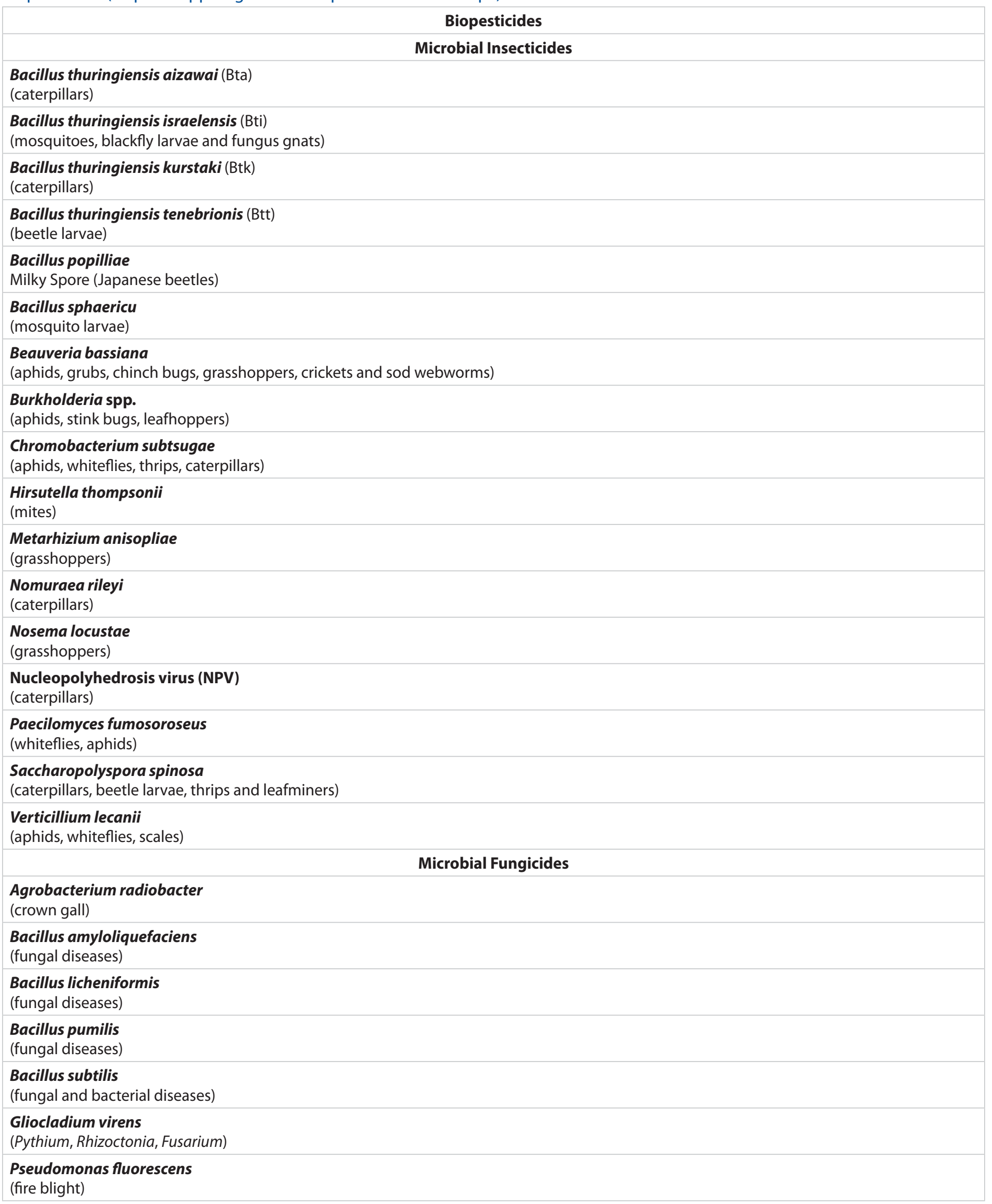




\section{Biopesticides}

Reynoutria sachalinensis

(Powdery mildew and Botrytis grey mold)

Streptomyces spp.

(Fusarium, damping off, Pythium, Phytophthora and fire blight)

Trichoderma spp.

(Pythium, Rhizoctonia, Fusarium, Cylindrocladium and Thielaviopsis)

Table 8. Association of Natural Biocontrol Producers practitioners that provide support services for biological control.

\begin{tabular}{|l|l|}
\hline \multicolumn{1}{|c|}{ Company } & \multicolumn{1}{c|}{ Services Provided } \\
\hline $\begin{array}{l}\text { BioTEPP Inc. } \\
\text { VIROSOFT CP4 - BioTEPP }\end{array}$ & $\begin{array}{l}\text { Produces environmentally friendly bio-pesticides to help reduce the spread of } \\
\text { chemical pesticides. }\end{array}$ \\
\hline $\begin{array}{l}\text { Buglady Consulting } \\
\text { http://www.bugladyconsulting.com/ }\end{array}$ & $\begin{array}{l}\text { Provides expert advice on pest management and plant health, focusing on } \\
\text { IPM and biological control. }\end{array}$ \\
\hline $\begin{array}{l}\text { LITSINGER IPM } \\
\text { Email: alitsinger80@yahoo.com }\end{array}$ & $\begin{array}{l}\text { Specializes in biological control with an organic emphasis on disease and pest } \\
\text { prevention for high-valued crops. }\end{array}$ \\
\hline $\begin{array}{l}\text { M3 Agriculture Technologies } \\
\text { https://m3agriculture.com/ }\end{array}$ & $\begin{array}{l}\text { Supports import, storage, transport, and aerial sterile insect and natural } \\
\text { enemy release using unmanned aircraft. }\end{array}$ \\
\hline $\begin{array}{l}\text { Parabug } \\
\text { Biocontrol by Drone | Parabug }\end{array}$ & $\begin{array}{l}\text { Uses unmanned aerial vehicles to apply commercially reared beneficial insects } \\
\text { to control agricultural pests. }\end{array}$ \\
\hline $\begin{array}{l}\text { The UAV-IQ Precision Ag. } \\
\text { https://www.uaviq.com }\end{array}$ & $\begin{array}{l}\text { Uses drones to release beneficial insects exactly when and where they're } \\
\text { needed to suppress pests. }\end{array}$ \\
\hline $\begin{array}{l}\text { The Quebec Institute for the Development of Ornamental } \\
\text { Horticulture (IQDHO) } \\
\text { Home - IQDHO }\end{array}$ & $\begin{array}{l}\text { Assists growers to determine their agroenvironmental profiles and set and } \\
\text { reach their IPM objectives. }\end{array}$ \\
\hline
\end{tabular}

